\title{
A topic-based sentence representation for extractive text summarization
}

\author{
Nikolaos Gialitsis \\ DIT, NKUA \\ nikolasyal@gmail.com
}

\author{
Nikiforos Pittaras \\ DIT, NKUA \\ IIT, NCSR-D \\ npittaras@di.uoa.gr
}

\author{
Panagiotis Stamatopoulos \\ DIT, NKUA \\ takis@di.uoa.gr
}

\begin{abstract}
We examine the effect of probabilistic topic model-based word representations, on sentence-based extractive summarization. We formulate the task of sentence selection as a binary classification problem, and we test a variety of machine learning algorithms, exploring a range of different settings for classification and modelling. A preliminary investigation via a wide experimental evaluation on the MultiLing 2015 MSS dataset illustrates that topicbased representations can prove beneficial to the extractive summarization process, compared to a TF-IDF baseline, with Quadratic Discriminant Analysis and Gradient Boosting providing the best results for micro and macro F1 score, respectively.
\end{abstract}

\section{Introduction}

In recent years, advances in the field of Natural Language Processing (NLP) have revolutionized the way machines are used to interpret humanwritten text. With the rapid accumulation of publicly available documents, from newspaper articles to social media posts, machine learning methods designed to automate data analysis are urgently needed. A problem that has been relevant since the dawn of NLP is the automatic summary extraction from a large corpus of text. The development of a consistent and time-efficient method of extractive summarization can assist journalists in their day to day tasks, as well as provide better tools for information retrieval.

Summaries need to be as brief as possible but must also capture the important elements of a text. This turns out to be a challenging task for any algorithm to carry out, since there is a virtually infi- nite number of documents that can exist, and each one of them can refer to a unique concept. Natural language is tricky for a computer to model; the absence or presence of a single word can shift the meaning of a whole sentence or even of a whole chapter. On the other hand, some words do not add any value to a sentence, the meaning is still the same even if we ignore them. To make matters even more complex, a word can be crucial for one article but of little importance to another.

Human brains have evolved to effectively detect complex patterns in text, to focus on the most important bits of a text while ignoring those that are less important. For a machine, the importance of word or a sentence is not obvious, as it needs to be programmed with a built-in way to assess it in any given context. For the purposes of summary extraction, an automatic summarizer needs to be able to compare words, or sentences via computational means, and announce those with the highest scores as the most relevant for a given document. The representation, aka. the method by which these similarity scores are assigned, is of critical importance to any summary extraction task.

When the representation is selected, the next step is training the model, that is, feeding the sentences represented as numerical sequences, to a machine learning procedure. If the representation and the dataset are suitable for the goal we are trying to accomplish, we can expect that the model will be able to predict which words or sentences are more important to a given document. Summing up all the sentences that the model considers to be important, results in a summary of the input text.

\section{Related work}

\subsection{Topic Modeling}




\subsubsection{Semantic Topics.}

Topics can be viewed as semantic groups that refer to a particular portion of reality. A document can refer to one or more distinct topics, which humans can often easily distinguish. For example, the words "fishing", "boat", "waves", have something in common; they are all affiliated with the sea. We can think of Sea as one topic, which contains these three words. However, topics are not always that identifiable and there can be broader or narrower topics. Resuming the previous example, alternatively, there can exist a topic on fishing , another one on boats and another one on ocean waves. Each one of them contains a number of words that are directly tied to that concept.

As demonstrated, there is no unique way to infer topics from an input document. It depends on the representation, the way that we measure the similarity scores between two words.It only makes sense that if two words are similar, they will have a high chance of belonging to the same topic.This statement derives from the distributional hypothesis in linguistics which proposes that words that occur in similar contexts tend to have similar meanings (Harris, 1954) However, we have to keep in mind that one word can also belong to one or more topics and that the number of topics in a document is also not known.

\subsubsection{Latent Dirichlet Allocation.}

Topic models can infer topics by observing the distribution of words across documents. This can be accomplished with Latent Dirichlet Allocation (LDA) (Steyvers and Griffiths, 2017; Blei, 2012), a generative statistical model that makes the hypothesis that there exists an underlying distribution of words,topics and documents, which generated the input text collection. Using probabilistic topic model jargon, the words of a document are called "observed variables", whereas the variables of the topic structure are called "hidden variables". Using an iterative process, the model estimates the posterior distribution of the hidden variables given the observed variables. However, the vast amount of topic structures that can exist result in exponential complexities of computation. For this reason, sampling-based algorithms have been developed, such as Gibbs sampling.

\subsubsection{Gibbs sampling}

In Gibbs sampling (Steyvers and Griffiths, 2017), a Markov chain (i.e., a sequence of random variables, each only dependent on the previous) is constructed, using samples from the distribution of hidden variables. The assignment of words to topics is sampled iteratively until the Markov chain converges to the target distribution. In the beginning of this procedure, each word is randomly assigned to a topic and in each subsequent iteration, the word-topic assignments are re-evaluated, which might result in words passing through multiple topics during the process.

\subsection{Vector Space Models}

Vector Space Model (VSM) approaches project the input to a $n$-dimensional vector representation, where the semantic similarity of the points is determined by their distance (e.g cosine, euclidean, etc.) in the projected vector space. Feature vector representations are widely used in Machine Learning tasks, e.g. for classification, clustering, etc. of a collection of input items (Turney and Pantel, 2010).

\subsubsection{Bag-of-words approaches}

A popular way to represent a set of documents as feature vectors has been the bag-of-words approach (Salton et al., 1975), where a sentence can be represented as a vector of word features. Each vector coordinate expresses word statistics, such as frequency or the Term Frequency-Inverse Document Frequency (TF-IDF) (Jones, 2004) value of a given word in the source texts. By mapping a word to its TF-IDF value, words receive a high weight when they appear often in the referenced document, but rarely in other documents of the set. The benefit of this approach is that it suppresses common words that appear in the majority of documents, without containing any semantic value for the task. It has been demonstrated that the approach can result in significant improvements over raw frequency approaches in a variety of information retrieval tasks. (Salton and Buckley, 1988).

\subsection{Extractive Summarization}

In extractive summarization, the summaries produced contain a subset of unmodified sentences contained in the original documents. Consequently, in these approaches, sentences, and not words, consist the units of feature selection. The pipeline of an extractive text summarizer is 
formed of three relatively independent tasks : (Rao and Gudivada, 2018)

1. Construction of an intermediate representation of the input text based on the key aspects of the text

2. Scoring the sentences based on the selected representation

3. Selection of the summary comprising of a number of sentences

Gupta and Lehal(2010) define a different division of tasks, which includes a pre-processing and a processing step. The pre-processing step also includes: sentence boundary identification, stopword elimination, and stemming. During the processing step, weights are assigned to specific sentence features by a feature-wise weighting mechanism, with the top ranked sentences being included in the final summary. In this study, we will follow the paradigm, proposed by Rao and Gudi$\operatorname{vada}(2018)$.

There are two types of representation-based approaches: 1) topic representations and indicator representations. A Topic representation transforms the text into an intermediate form and interprets the topic(s) discussed in the text. The techniques used for this, differ in terms of their complexity, and are divided into frequency-driven approaches, topic word approaches, latent semantic analysis and Bayesian topic models. Indicator representation describes every sentence as a list of formal features (indicators) of importance such as sentence length, position in the document, or having certain phrases; the use of indicators was demonstrated by J et al.(2008).

\subsubsection{Sentence-based summarization}

In contrast to bag-of-words representations that suffer from the curse of dimensionality (Bellman, 1958), more sophisticated recent approaches produce sentence vectors in a lower dimensional space, such as a latent-topic space. Many such these methods utilize topic clusters in order to locate the centroids (or medoids in non-euclidean spaces) that best represent the sentences in the topics. Then the score of each sentence is assigned in respect to its distance from the clusters' representatives. For example, Thomas et al.(2015) used a graph-based procedure where each node of the graph represents a sentence and the edges' weights reflect the similarity between the connected nodes. Next, a PageRank/TextRank algorithm is applied

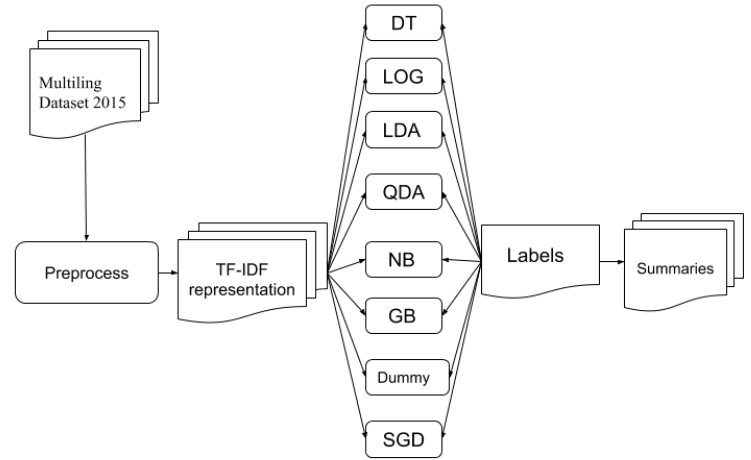

Figure 1: The pipeline for the TF-IDF-based extractive summarization

to extract the sentence representatives based on the graph centrality. In another topic-based approach,featured by Vicente et al. (2015) Principal Component Analysis (PCA) was used to project the sentences into a lower-dimension space. The principal components are then evaluated and the sentences with the highest scores get selected to appear in the summary.

\subsubsection{Contributions}

There are some limitations with the majority of the existing topic-based summarization methods. First, they work directly in the sentence space and the term-topic information embedded in the sentences is ignored.

In this study, we combine the simplicity of word-level approaches with the power of probabilistic topic models; instead of limiting word information to a single value (e.g. frequency or TFIDF weight), we model sentences with word-level topic assignments. This approach is supported by a clear and rigorous probabilistic interpretation (rather than some ad-hoc sentence-level aggregation of a multitude of unrelated scores) and produces rich, semantic sentence-level representations.

\section{Proposed Method}

\subsection{Binary classification modelling}

Extractive summarization can be modelled as a binary classification problem, where one class represents the sentences to be included in the summary, and the other one the sentences that should be ignored. More formally, a document comprised of $N$ sentences $S=\left\{s_{i}\right\}, i \in\{1, \ldots N\}$ is 


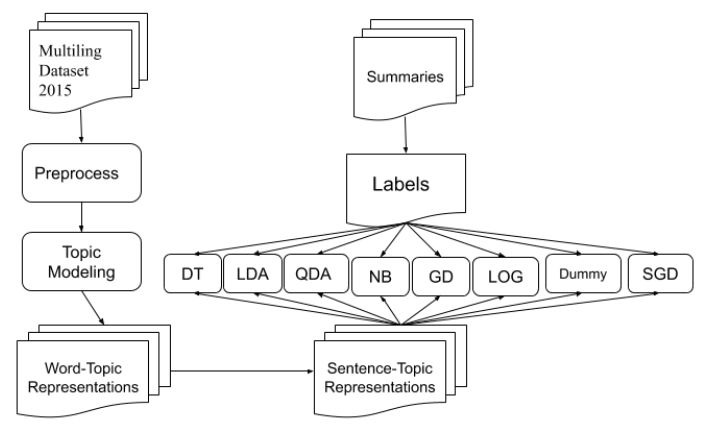

Figure 2: pipeline for topic-modeling based extractive summarization

transformed to a subset of $M$ sentence summaries $O=\left\{o_{j}\right\}, o_{j} \in S, j \in\{1 \ldots M\}$ via a classifier that maps each sentence to a binary label (denoted inclusion in the summary or not). The classification model should select $O$ such that the concatenation of its sentences should produce a coherent, non-reductive and readable summary.

In this study, we tackle classification as a supervised learning procedure; it is necessary to have a set of ground truth sentences, that is, sentences that are indeed valid summaries of input documents. Such data (commonly referred to as "golden" summaries) are manually compiled by humans, who are considered the best summarizers (Genest et al., 2009); if a human reader can not differentiate between a human summarizer and an automatic summarizer, that means that the extractive model is optimal. Using the input documents and ground truth data, the classification system can facilitate learning using input sentence features towards a saliency detection model that implements sentence selection towards extractive summarization. We detail this process in the next sections.

\subsection{Topic-based Sentence Extraction}

In our approach of extractive summarization, we utilize the topics' information in word-level feature vector representations using an LDA-based topic model with Gibbs sampling.

The intuition behind our proposed method follows two statements:(1) the significance of a word is reflected by its contribution to a set of semantic topics (2) the significance of a sentence is reflected by patterns in its words-topics contributions.

For the purpose of formality we provide the mathematical description of the proposed method.

Given a finite set of semantic topics $T=$ $\left\{T_{1}, T_{2}, \ldots T_{|T|}\right\}$ over the documents' space $D$, a set of sentences per document $S_{D_{i}}=$ $\left\{s_{1}, s_{2}, \ldots, s_{k}\right\}$, and a set of words per sentence $W_{S_{D_{i}}}=\left\{w_{1}, w_{2}, \ldots w_{n}\right\}$, we define the wordtopics contribution function of a word $w$ as:

$$
C(w)=\left[p\left(w, T_{1}\right), p\left(w, T_{2}\right), \ldots p\left(w, T_{|T|}\right)\right]
$$

where the vector $C(w)$ is the contribution of the word $w$ to the topics set $T$ and $p\left(w, T_{i}\right)$ is the probability of $w$ being generated by the topic $T_{i} \in T$ (after the topic model has inferred the posterior probability distributions), as defined by LDA's term-topic distribution. In simpler terms, this probability is computed using:

$$
p\left(w, T_{i}\right)=\frac{N\left(w, T_{i}\right)}{N\left(T_{i}\right)}
$$

where $N\left(w, T_{i}\right)$ and $N\left(T_{i}\right)$ are the number of occurences of $w$ in $T_{i}$ and the total number of word occurences in $T_{i}$, respectively.

Further, normalization is applied over the contributions of each word vector, in order to project the values into the $\{0,1\}$ interval, dividing each value by the maximum value in each vector :

$$
C_{i}(w)=\frac{C_{i}(w)}{\max (C(w))} \forall i \in\{1,|T|\}
$$

where $\max (C(w)) \neq 0$

After all word-topic contributions have been calculated, each sentence $s=\left\{w_{1}, w_{2}, \ldots, w_{n}\right\}$ is represented by the vector

$$
C(s)=\left[C\left(w_{1}\right), C\left(w_{2}\right), \ldots, C\left(w_{n}\right)\right],
$$

effectively transforming an input set of sentences $S=\left\{s_{1}, s_{2}, . . s_{k}\right\}$ into the multi-dimensional vector

$$
S^{\prime}=\left[C\left(s_{1}\right), C\left(s_{2}\right), \ldots, C\left(s_{k}\right)\right]
$$

Since most machine learning algorithms work with data of equal dimensionality, we apply padding to enforce a uniform dimension accross sentences. In zero-padding, the smaller-sized vectors are appended with zeros until all vectors have the same number of dimensions. Since there can be sentences with different dimensions in the documents examined, we implement zero-padding, in 
order for the elements of $S^{\prime}$ in equation (5) to become uniform.

\section{Experiments}

\subsection{Dataset and Preprocessing}

We use the Multiling 2015 dataset for singledocument summarization (Giannakopoulos et al., 2015) ${ }^{1}$. The dataset is constructed by the MultiLing community (Conroy et al., 2015) from wikipedia pages, using articles annotated by human-curated summaries. It consists of 40 languages, spanning 30 documents and summary sets - in our work, we restrict the evaluation to the English language, i.e. work with the 30 English documents provided.

We modify the dataset in order to align it with the extractive summarization setting (as the provided summaries are not purely document sentences). First, the ground truth is modified, labelling input source sentences with a label $l \in$ 0,1 ( 1 if the sentence should be included in the summary, else 0 ). This is computed by measuring the similarity of each source sentence with each human-authored summary for the document, in terms of common n-grams. I.e, each humanauthored sentence $g_{i}$ is assigned to a maximally similar source sentence $s_{j}$. Stopword filtering is applied prior to this process, and each source sentence is assigned to at most one ground truth sentence.

Additionally, since the dataset used contains very unbalanced classes - the grand majority (with a ratio approximately 13 to 1 ) belonging to class 0 , i.e. the class for sentences that should not be included in the summaries. To alleviate this, we employ an oversampling scheme. To limit the bias towards class 0 during the training phase of our model, we implemented oversampling, by repeating the sentences belonging to class 1 a fixed number of times arriving at a $2: 1$ negative to positive ratio, at most. This way, a classifier that always predicts dominant label (in this case 0) has suboptimal performance.

Also, all letters were converted to lower-case in order for the model not to differentiate between words in the beginning and in the middle of sentences, such as "apples" and "Apples". In addition, stop words were also removed from the vocabulary to limit its size, without significant loss

\footnotetext{
${ }^{1}$ http://multiling.iit.demokritos.gr/ pages/view/1516/multiling-2015
}

\begin{tabular}{|l|c|c|}
\hline & train & test \\
\hline mean num. sentences & 233 & 184.9 \\
\hline mean summ. sentences & 77.9 & 13.5 \\
\hline mean num. words & 25.5 & 22.8 \\
\hline sample sentences & 6990 & 5546 \\
\hline
\end{tabular}

Table 1: Multiling 2015 single-document summarization dataset characteristics.

of information.

Other preprocessing tasks such as stemming was also explored; however, they did not have a significant effect on the classification performance. After these steps, we end up with the final version of the dataset which is described in detail in table 1.

\subsection{Evaluation}

We use the provided training and test dataset portion to train and evaluate the produced classifiers. The evaluation is performed in terms of micro and macro F-measure; the former is calculated by counting the total true positives, false negatives and false positives while the macro-averaged variant calculates metrics for each label, and finds their unweighted mean (i.e., not considering label imbalance). Additionally, we compare the predicted summaries with the ground-truth as described in section 4.1, using the Rouge metric to assess performance (Lin, 2004) ${ }^{2}$. Rouge scores reflect the overlap of $\mathrm{n}$-grams between the groundtruth and the predicted summaries.

\subsection{TF-IDF Sentence Classification}

As a baseline model, we also implemented a TFIDF representation of the input dataset. The TFIDF scores for each word-document pair are calculated and each sentence is represented by the vector of the tf-idf values of the words it contains. For example, a sentence with $N$ words results in a $N_{w^{-}}$dimensional vector, where $N_{w}$ is the number of words in the sentence.

The pipeline for sentences classification using the tf-idf approach is summarized schematically in Figure 1.The scikit-learn v0.21.3 machine learning library ${ }^{3}$ is used for building and training the models.

\footnotetext{
${ }^{2}$ https://pypi.org/project/py-rouge/

${ }^{3}$ https://scikit-learn.org/stable/ index.html
} 


\begin{tabular}{|l|l|l|l|l|l|l|l|l|l|}
\hline Metric & DT & KNN & GB & NB & LDA & QDA & Dummy & LOG & SGD \\
\hline macro-f1 & 0,497 & 0,511 & 0,514 & 0,514 & $\mathbf{0 , 5 2 7}$ & 0,080 & 0,452 & $\mathbf{0 , 5 2 7}$ & 0,481 \\
\hline micro-f1 & 0,898 & 0,900 & 0,918 & 0,911 & 0,903 & 0,083 & 0,643 & 0,883 & $\mathbf{0 , 9 2 7}$ \\
\hline
\end{tabular}

Table 2: TF-IDF sentence classification results.

\subsection{Topic Modeling-based Classification of sentences}

For the production of the topics and the topicvectors we used MALLET, a Java framework for various common tasks in NLP, including topicmodeling (McCallum, 2002). Using this tool, we inferred topics over the corpus of the documents in the training set. We subsequently represent firstly the words, and lastly the sentences, of the documents in the training set by their topiccontributions as described in section 3.2. By default, MALLET ignores all 1-letter and 2-letter words. Additionally, we use the NLTK english stop-words list for stop-word filtering ${ }^{4}$.

We test the trained topic model by extracting word and sentence-level probabilistic vector representations from the test set. Any word in the test set not present in the training set, is represented as a zero-vector of topic-contributions.

The pipeline for sentence classification using the topics-based approach can be visualized in figure 2 and is outlined below:

- Infer k topics using MALLET's topic model from the training set

- Represent each sentence in the training set using the equation (4).

- Train a classifier on the topics-represented training set

- Represent each sentence in the test set using the trained model from Step 1

- Predict the labels in the represented test set

- Evaluate the classifier using the micro and macro f-measures

\section{Results and Discussion}

\subsection{Classification Results}

The experimental results of the classification on the Multiling Dataset, evaluated with the microf1 and macro-f1 scores are displayed in tables 2 and 4, for the TF-IDF representation and the topicbased representation, respectively. Baseline results using a simple rule-based classifier (Dummy)

\footnotetext{
${ }^{4}$ https://gist.github.com/sebleier/ 554280
}
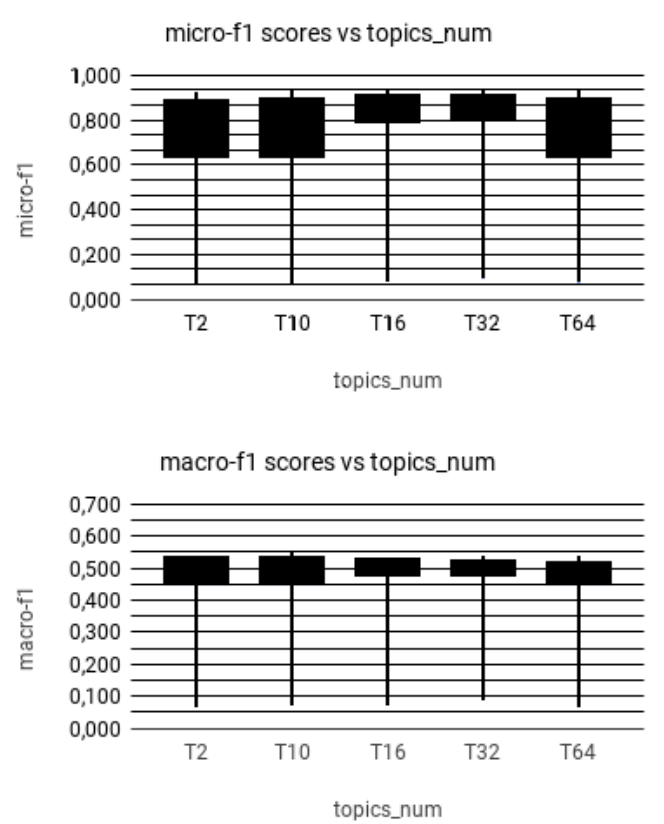

Table 3: Comparison of the micro (top) and macro (bottom) f1 performance of topic modeling, based based on the selection of the number of topics

are also reported, generating predictions with respect to the training set's class distribution - it is thus not influenced by the representation. Dummy gives a micro-f1 score of 0.643 and a macro-f1 score of 0.452 .

For TF-IDF, the best macro-f1 score recorded is 0.527 achieved by the Linear Discriminant Analysis (LDA) and Logistic Regression Classifiers (LOG) and the best micro-f1 score is 0.927 , given by the Stochastic Gradient Descent Classifier (SGD). TF-IDF achieves significantly better classification results than Dummy, improving micro-f1 by $28 \%$ and macro-f1 by $7 \%$, verifying the effectiveness of simple bag-of-word approaches.

For the topic-based representation of sentences, we ran the topic model with a different numbers of topics $k$ on each run, and we trained various classifiers for the task. One major limitation of topic-modeling is that the number of topics needs to be determined experimentally. In order to make an informed decision on $k$, we examined candi- 


\begin{tabular}{|l|l|l|l|l|l|l|l|l|l|}
\hline \multicolumn{10}{|c|}{ MICRO F-MEASURE } \\
\hline Topics & DT & KNN & GB & NB & LDA & QDA & Dummy & $\underline{\text { LOG }}$ & SGD \\
\hline 2 & 0,885 & 0,812 & 0,884 & $\mathbf{0 , 9 2 2}$ & 0,879 & 0,102 & 0,643 & 0,754 & 0,073 \\
\hline 10 & 0,889 & 0,752 & 0,895 & 0,078 & 0,873 & 0,175 & 0,643 & 0,782 & $\mathbf{0 , 9 2 7}$ \\
\hline $\mathbf{1 6}$ & 0,889 & 0,802 & 0,908 & 0,080 & 0,884 & $\mathbf{0 , 9 2 8}$ & 0,643 & 0,800 & 0,927 \\
\hline 32 & 0,894 & 0,864 & 0,909 & 0,093 & 0,866 & $\mathbf{0 , 9 2 7}$ & 0,643 & 0,813 & $\mathbf{0 , 9 2 7}$ \\
\hline 64 & 0,892 & 0,872 & 0,916 & 0,149 & 0,861 & $\mathbf{0 , 9 2 7}$ & 0,643 & 0,856 & 0,073 \\
\hline \hline mean & $\mathbf{0 , 8 9 0}$ & 0,820 & $\mathbf{0 , 9 0 2}$ & 0,264 & $\mathbf{0 , 8 7 3}$ & 0,612 & 0,643 & 0,801 & 0,585 \\
\hline std & $\mathbf{0 , 0 0 3}$ & 0,044 & $\mathbf{0 , 0 1 1}$ & 0,330 & $\mathbf{0 , 0 0 8}$ & 0,387 & 0,000 & 0,034 & 0,418 \\
\hline \multicolumn{8}{|c|}{ MACRO F-MEASURE } \\
\hline Topics & DT & KNN & $\mathbf{G B}$ & NB & LDA & QDA & Dummy & LOG & SGD \\
\hline 2 & $\mathbf{0 , 5 3 5}$ & 0,524 & $\mathbf{0 , 5 3 5}$ & 0,489 & 0,531 & 0,101 & 0,452 & 0,513 & 0,068 \\
\hline $\mathbf{1 0}$ & 0,532 & 0,506 & $\mathbf{0 , 5 4 6}$ & 0,073 & 0,535 & 0,172 & 0,452 & 0,520 & 0,481 \\
\hline 16 & 0,528 & 0,513 & $\mathbf{0 , 5 3 4}$ & 0,076 & 0,533 & 0,505 & 0,452 & 0,517 & 0,481 \\
\hline 32 & $\mathbf{0 , 5 3 7}$ & 0,517 & 0,531 & 0,091 & 0,522 & 0,481 & 0,452 & 0,521 & 0,481 \\
\hline 64 & 0,517 & 0,512 & 0,516 & 0,149 & $\mathbf{0 , 5 3 9}$ & 0,484 & 0,452 & 0,527 & 0,068 \\
\hline \hline mean & 0,530 & 0,514 & $\mathbf{0 , 5 3 2}$ & 0,176 & $\mathbf{0 , 5 3 2}$ & 0,349 & 0,452 & 0,520 & 0,316 \\
\hline std & $\mathbf{0 , 0 0 7}$ & $\mathbf{0 , 0 0 6}$ & $\mathbf{0 , 0 1 0}$ & 0,159 & $\mathbf{0 , 0 0 6}$ & 0,175 & 0,000 & $\mathbf{0 , 0 0 5}$ & 0,202 \\
\hline
\end{tabular}

Table 4: Topic modeling results in micro and macro F1 score.

date values for the number of topics, visualized in box-plots presented in table 3. By analyzing table 4 and the box-plots, we concluded that a satisfactory number of topics is 10 for this particular task, as for this $k$, the Gradient Boosting Classifier (GB) records the highest macro-f1 score. Our decisions are biased towards the macro-f1 instead of the micro-f1 score, since even after the over-sampling of the dataset, the classes are still heavily imbalanced. In addition, we are mostly interested in the sentences that should be included in the summary, which belong to the smaller class. One thing to note, is that as the topic dimension increases, the macro-f1 performance of the Quadratic Discriminant Analysis classifier increases rapidly between Topics 2 and Topics 32 where it reaches a plateau at macro $\mathrm{F} 1 \approx 0.48$.

Topic-modeling improves on the measures of TF-IDF and Dummy, with a 0.928 micro-f1 score given by the Quadratic Discriminant Analysis (Topics 16) and a 0.546 macro-f1 score given by Gradient Boosting Classifier(Topics 10) resulting in a $3.6 \%$ increase in performance, in comparison with the TF-IDF macro-f1 score. The worstperforming classifiers for the selected number of topics are the Naive Bayes (NB) and Quadratic Discriminant Analysis classifiers.

Finally, considering across-topics averages, SGD, QDA and NB appear to be the least stable configurations, while GB, LDA and DT are among the top performers.

\subsection{Rouge scores}

The rouge scores of the summaries produced by the representation-classifier combinations are displayed in tables 5 and 6 . Even though we observed considerable differences in the classification phase between the two representations overall, the final rouge scores are more similar than expected. Bold values correspond to the maximum f-measures for each rouge-metric.

For the TF-IDF, the highest rouge-scores across all classifiers were given by the Quadratic Discriminant Analysis (QDA), while for the Topicsrepresentation, the highest values were recorded by the Naive Bayes Classifier (NB) and Gradient Boosting (GB). The TF-IDF representation results in slightly better rouge- 1 to rouge- 4 scores while the Topics-based representation produces better rouge-l and rouge-w scores.

\section{Conclusions}

In this work, we investigated the contribution of topic-based sentence classification to extractive summarization. We examined a variety of configurations for topic modeling by examining a wide range of topics, along with a set of different, diverse classification algorithms. A subsequent large-scale evaluation was performed us- 


\begin{tabular}{|c|c|c|c|c|c|c|c|}
\hline \multirow[t]{2}{*}{ CLASSIFIER } & & & & \multicolumn{4}{|l|}{ METRIC } \\
\hline & & rouge-1 & rouge- 2 & rouge-3 & rouge-4 & rouge-I & rouge-w \\
\hline \multirow{3}{*}{ KNN } & recall & 0,226 & 0,042 & 0,013 & 0,007 & 0,170 & 0,034 \\
\hline & precision & 0,307 & 0,056 & 0,017 & 0,008 & 0,232 & 0,127 \\
\hline & f1 & 0,245 & 0,046 & 0,014 & 0,007 & 0,186 & 0,051 \\
\hline \multirow{3}{*}{ LDA } & recall & 0,127 & 0,025 & 0,008 & 0,003 & 0,096 & 0,019 \\
\hline & precision & 0,161 & 0,036 & 0,017 & 0,011 & 0,120 & 0,065 \\
\hline & f1 & 0,136 & 0,027 & 0,010 & 0,004 & 0,103 & 0,029 \\
\hline \multirow{3}{*}{ GB } & recall & 0,164 & 0,032 & 0,008 & 0,004 & 0,132 & 0,026 \\
\hline & precision & 0,258 & 0,060 & 0,019 & 0,013 & 0,199 & 0,113 \\
\hline & f1 & 0,186 & 0,038 & 0,010 & 0,005 & 0,149 & 0,040 \\
\hline \multirow{3}{*}{ LOG } & recall & 0,153 & 0,031 & 0,009 & 0,003 & 0,115 & 0,023 \\
\hline & precision & 0,184 & 0,038 & 0,012 & 0,006 & 0,136 & 0,071 \\
\hline & f1 & 0,162 & 0,034 & 0,010 & 0,004 & 0,122 & 0,034 \\
\hline \multirow{3}{*}{ QDA } & recall & 0,365 & 0,106 & 0,047 & 0,026 & 0,264 & 0,056 \\
\hline & precision & 0,364 & 0,106 & 0,047 & 0,027 & 0,264 & 0,140 \\
\hline & f1 & 0,365 & 0,106 & 0,047 & 0,027 & 0,264 & 0,080 \\
\hline \multirow{3}{*}{ Dummy } & recall & 0,344 & 0,076 & 0,029 & 0,014 & 0,242 & 0,050 \\
\hline & precision & 0,345 & 0,076 & 0,028 & 0,014 & 0,243 & 0,125 \\
\hline & f1 & 0,344 & 0,076 & 0,029 & 0,014 & 0,242 & 0,071 \\
\hline \multirow{3}{*}{ NB } & recall & 0,208 & 0,034 & 0,006 & 0,001 & 0,148 & 0,029 \\
\hline & precision & 0,232 & 0,037 & 0,006 & 0,001 & 0,164 & 0,082 \\
\hline & f1 & 0,216 & 0,035 & 0,006 & 0,001 & 0,154 & 0,042 \\
\hline \multirow{3}{*}{ DT } & recall & 0,280 & 0,043 & 0,010 & 0,003 & 0,207 & 0,041 \\
\hline & precision & 0,323 & 0,045 & 0,010 & 0,003 & 0,239 & 0,122 \\
\hline & f1 & 0,292 & 0,044 & 0,010 & 0,003 & 0,216 & 0,060 \\
\hline \multirow{3}{*}{ SGD } & recall & $\overline{0,000}$ & 0,000 & 0,000 & 0,000 & 0,000 & 0,000 \\
\hline & precision & 0,000 & 0,000 & 0,000 & 0,000 & 0,000 & 0,000 \\
\hline & $\mathrm{f} 1$ & 0,000 & 0,000 & 0,000 & 0,000 & 0,000 & 0,000 \\
\hline
\end{tabular}

Table 5: TF-IDF Rouge Scores

\begin{tabular}{|c|c|c|c|c|c|c|c|}
\hline \multirow[t]{2}{*}{ CLASSIFIER } & & & & METRIC & & & \\
\hline & & rouge-1 & rouge-2 & rouge-3 & rouge-4 & rouge-I & rouge-w \\
\hline \multirow{3}{*}{ KNN } & recall & 0.326 & 0.06 & 0.019 & 0.01 & 0.238 & 0.048 \\
\hline & precsion & 0.332 & 0.062 & 0.019 & 0.01 & 0.241 & 0.122 \\
\hline & f1 & 0.328 & 0.061 & 0.019 & 0.01 & 0.239 & 0.069 \\
\hline \multirow{3}{*}{ LDA } & recall & 0.365 & 0.105 & 0.046 & 0.026 & 0.268 & 0.057 \\
\hline & precision & 0.332 & 0.063 & 0.018 & 0.009 & 0.236 & 0.118 \\
\hline & f1 & 0.327 & 0.062 & 0.018 & 0.009 & 0.232 & 0.066 \\
\hline \multirow{3}{*}{ GB } & recall & 0.334 & 0.069 & 0.025 & 0.015 & 0.242 & 0.049 \\
\hline & precision & 0.361 & 0.105 & 0.046 & 0.026 & 0.265 & 0.14 \\
\hline & f1 & 0.361 & 0.104 & 0.046 & 0.026 & 0.265 & 0.08 \\
\hline \multirow{3}{*}{ LOG } & recall & 0.362 & 0.102 & 0.045 & 0.025 & 0.267 & 0.056 \\
\hline & precision & 0.339 & 0.069 & 0.025 & 0.015 & 0.245 & 0.125 \\
\hline & f1 & 0.336 & 0.069 & 0.025 & 0.015 & 0.243 & 0.071 \\
\hline \multirow{3}{*}{ QDA } & recall & 0.305 & 0.064 & 0.022 & 0.012 & 0.221 & 0.045 \\
\hline & precision & 0.362 & 0.101 & 0.045 & 0.025 & 0.267 & 0.141 \\
\hline & f1 & 0.361 & 0.101 & 0.045 & 0.025 & 0.267 & 0.08 \\
\hline \multirow{3}{*}{ Dummy } & recall & 0.344 & 0.076 & 0.029 & 0.014 & 0.242 & 0.05 \\
\hline & precision & 0.345 & 0.076 & 0.028 & 0.014 & 0.243 & 0.125 \\
\hline & f1 & 0.344 & 0.076 & 0.029 & 0.014 & 0.242 & 0.071 \\
\hline \multirow{3}{*}{ NB } & recall & 0.313 & 0.063 & 0.021 & 0.013 & 0.228 & 0.046 \\
\hline & precision & 0.364 & 0.104 & 0.046 & 0.026 & 0.268 & 0.142 \\
\hline & f1 & 0.364 & 0.104 & 0.045 & 0.026 & 0.267 & 0.081 \\
\hline \multirow{3}{*}{ DT } & recall & 0.363 & 0.105 & 0.046 & 0.025 & 0.265 & 0.056 \\
\hline & precision & 0.33 & 0.065 & 0.022 & 0.013 & 0.241 & 0.125 \\
\hline & f1 & 0.319 & 0.064 & 0.022 & 0.013 & 0.232 & 0.067 \\
\hline \multirow{3}{*}{ SGD } & recall & 0.323 & 0.062 & 0.018 & 0.009 & 0.23 & 0.046 \\
\hline & precision & 0.331 & 0.067 & 0.022 & 0.012 & 0.238 & 0.124 \\
\hline & $\mathrm{f} 1$ & 0.312 & 0.065 & 0.022 & 0.012 & 0.226 & 0.066 \\
\hline
\end{tabular}

Table 6: Topic modeling Rouge Scores ing micro-f1 and macro-f1 scores. Based on the trained models, we produced summaries for the input documents and we compared them with the ground-truth using several Rouge-metrics. As a baseline, we also implemented a TF-IDF representation of sentences, which follows a traditional bag-of-words weighted approach.

Initial results of this early study show that topicmodeling can be beneficial for sentence classification, as it outperforms the TF-IDF representation, as illustrated by the micro and macro f1 scores in our experiments, albeit this not being the case for the Rouge-based evaluation. We demonstrated that the topics-based approach can easily compete with the TF-IDF approach and shows promise in extractive summarization. Careful task-specific adjustments need to be made however, as the results in the summary evaluation (using Rouge) appear underwhelming compared to those in the classification phase.

In the future, more sophisticated methods such as Principal Component Analysis(PCA) (Jolliffe, 2011) or Linear Semantic Analysis(LSA) (Landauer et al., 1998) can be applied on the presented framework of topics-based sentence representation, in order to project the word-topic vectors into lower-dimensional spaces.

Additionally, more adaptive topic modelling approaches could be applied, removing the need for pre-determined topic specification,(Steyvers and Griffiths, 2017). Moreover, Neural Network classification architectures can be explored, in addition to the set of classifiers we already tested on the dataset. A-priori knowledge on words, phrases and sentences from external sources (e.g. knowledge bases such as Wordnet (A. Miller et al., 1991)) could also prove beneficial for the training phase of the machine-learning models. Finally, future work will take order / target summary length into account, making our results comparable to other systems tackling the Multiling2015 dataset and the state of the art. 


\section{References}

A. Miller, G., Beckwith, R., Fellbaum, C., Gross, D., and J. Miller, K. (1991). Introduction to WordNet: An On-line Lexical Database*. 3.

Bellman, R. (1958). Dynamic programming and stochastic control processes. Information and Control, 1(3):228 - 239.

Blei, D. M. (2012). Probabilistic topic models. Communications of the ACM, 55(4):77.

Conroy, J. M., Kubina, J., Rankel, P. A., and Yang, J. S. (2015). Multilingual Summarization and Evaluation Using Wikipedia Featured Articles, chapter Chapter 9, pages 281-336.

Genest, P.-E., Lapalme, G., and Yousfi-Monod, M. (2009). Hextac: the creation of a manual extractive run.

Giannakopoulos, G., Kubina, J., Conroy, J., Steinberger, J., Favre, B., Kabadjov, M., Kruschwitz, U., and Poesio, M. (2015). MultiLing 2015: Multilingual Summarization of Single and MultiDocuments, On-line Fora, and Call-center Conversations. In Proceedings of the 16th Annual Meeting of the Special Interest Group on Discourse and Dialogue, pages 270-274, Prague, Czech Republic. Association for Computational Linguistics.

Gupta, V. and Lehal, G. S. (2010). A Survey of Text Summarization Extractive Techniques. Journal of Emerging Technologies in Web Intelligence, 2(3).

Harris, Z. S. (1954). Distributional Structure. WORD, 10(2-3):146-162.

J, J., Pingali, P., Varma, V., J, J., Pingali, P., and Varma, V. (2008). Sentence Extraction Based Single Document Summarization.

Jolliffe, I. (2011). Principal Component Analysis. In Lovric, M., editor, International Encyclopedia of Statistical Science, pages 1094-1096. Springer Berlin Heidelberg, Berlin, Heidelberg.

Jones, K. S. (2004). A statistical interpretation of term specificity and its application in retrieval. page 9.

Landauer, T. K., Foltz, P. W., and Laham, D. (1998). An introduction to latent semantic analysis. Discourse Processes, 25(2-3):259-284.

Lin, C.-Y. (2004). ROUGE: A Package for Automatic Evaluation of Summaries. page 8.

McCallum, A. K. (2002). MALLET: A Machine Learning for Language Toolkit.

Rao, C. and Gudivada, V. (2018). Computational Analysis and Understanding of Natural Languages: Principles, Methods and Applications. Handbook of Statistics. Elsevier Science.
Salton, G. and Buckley, C. (1988). Term-weighting approaches in automatic text retrieval. Information Processing \& Management, 24(5):513-523.

Salton, G., Wong, A., and Yang, C.-S. (1975). A vector space model for automatic indexing. Communications of the ACM, 18(11):613-620.

Steyvers, M. and Griffiths, T. (2017). Probabilistic Topic Models. page 15.

Thomas, S., Beutenmüller, C., de la Puente, X., Remus, R., and Bordag, S. (2015). ExB Text Summarizer. In Proceedings of the 16th Annual Meeting of the Special Interest Group on Discourse and Dialogue, pages 260-269, Prague, Czech Republic. Association for Computational Linguistics.

Turney, P. D. and Pantel, P. (2010). From Frequency to Meaning: Vector Space Models of Semantics. Journal of Artificial Intelligence Research, 37:141-188.

Vicente, M., Alcón, O., and Lloret, E. (2015). The University of Alicante at MultiLing 2015: approach, results and further insights. In Proceedings of the 16th Annual Meeting of the Special Interest Group on Discourse and Dialogue, pages 250-259, Prague, Czech Republic. Association for Computational Linguistics. 